\title{
Respiratory delivery of favipiravir-tocilizumab combination through mucoadhesive protein-lipidic nanovesicles: prospective therapeutics against COVID-19
}

\author{
Vikram Thakur ${ }^{1} \cdot$ Radha Kanta Ratho $^{1} \cdot$ Jiban Jyoti Panda $^{2}$
}

Received: 11 January 2021 / Accepted: 2 March 2021 / Published online: 17 March 2021

(C) Indian Virological Society 2021

\begin{abstract}
Coronavirus disease 19 (COVID-19) is the prime global health concern of the year 2020. Infecting more than 112 million individuals so far, this pandemic has already reported more than 2.4 million deaths around the world. With such high infectivity and mortality, effective treatment intervention is the need of the hour. The integration of medical science with nanotechnology may solve the current problem by exploring collective benefits. In this manuscript, we theoretically proposed the duo-combination of an approved antiviral i.e. favipiravir along with an immunomodulator i.e. tocilizumab loaded in protein-lipid nanovesicles as an effective anti-COVID-19 therapeutic. This proposed nanomedicine delivered through the respiratory mode may enhance the effectiveness of the antiviral and help in restricting the virus and associated complications, utilizing both anti-viral activity and immunomodulation in COVID-19 patients. This proposed nanomedicine could be an effective treatment modality for the severe acute respiratory syndrome- coronavirus-2 (SARS-CoV-2) infected patients.
\end{abstract}

Radha Kanta Ratho

rathopgi@yahoo.com

$\bowtie$ Jiban Jyoti Panda

jyoti@inst.ac.in

Vikram Thakur

vik5atif@gmail.com

1 Department of Virology, Post Graduate Institute of Medical Education and Research, PGIMER, Sector-12, Chandigarh 160012, India

2 Chemical Biology Unit, Nanotherapeutics Lab, Institute of Nano Science and Technology, (INST), Phase-10, Sector-64, Mohali, Punjab 160062, India
Keywords SARS-CoV-2 · COVID-19 · Nanomedicine · Respiratory $\cdot$ Nanovesicles $\cdot$ Favipiravir

\section{Introduction}

Since the day of inception as of now there are more than 112 million COVID-19 cases with more than 2.4 million deaths reported over 213 countries [1]. SARS-COV-2 with an R。 value of 2.5 (range 1.8-3.6) has way surpassed SARS 2002 (0.19-1.08) and deadly Ebola 2014 (1.5-1.9) in terms of the total number of infections and the resultant number of deaths [2-4]. $\mathrm{R}$ 。 is defined as the average number of people infected by one infectious individual during their infectious period. Looking into its infectivity and transmissibility, the World Health Organization (WHO) has declared the outbreak on March 11, 2020, a pandemic [5]. The understanding of COVID-19 is changing each day in regards to the viral entity, mode of transmission, virulent variants, varied pulmonary and extra-pulmonary manifestations, and molecular pathogenesis. The nationwide lockdown in a phased manner, social distancing, use of masks, frequent hand washing, and sanitization seem to be very effective in curtailing transmission of SARS-CoV-2 in the community. Presently following the lockdown scenario and with the ease of travel restrictions, the community spread of COVID-19 remains to be a challenge. Thus, the development of an effective vaccine or antiviral drug is the need of the hour to tackle the evolving SARS-CoV-2 variants. Amidst the alternative therapeutic modalities included are supportive oxygen therapy and plasma therapy with some promise. The development of herd immunity is essential but needs time to develop [6]. Also, researchers around the world are burning their mid-night oils to find a therapeutic vaccine or cure for the disease and the recent 
advancement and progress in antiviral and vaccine research worldwide is commendable. Solidarity trials for COVID19 have been organized by the WHO for determining the safety and efficacy of drugs like Remdesivir, and Interferon-beta (IFN- $\beta$ ) [7]. To date, approval has been granted to Dexamethasone (UK, and Japan), Avigan (China, and Italy), and Remdesivir (Japan) as a part of COVID-19 treatment. SARS-CoV-2 recombinant nanoparticle-based vaccines like NVX-CoV2373 (trimeric spike glycoprotein and Matrix-M1 adjuvant) showed enhanced CD4 + T-cell response post 35 days of vaccine administration [8]. Also, Ferritin nanoparticles-based subunit vaccine projecting SARS-CoV-2 spikes (S-Fer) has been shown to enhance the elicitation of neutralizing antibodies on single-dose vaccination [9]. So far only two COVID-19 vaccines developed by Pfizer-BioNTech and Moderna are approved by FDA under Emergency Use Authorization (EUA) [10]. Interestingly, both the Moderna and BioNTech vaccine candidates are mRNA vaccines encapsulated in lipid nanoparticles. As per COVID-19 vaccine draft dated 16 February 2021, 69 vaccine candidates are in the clinical phase, whereas 181 candidates are in pre-clinical trials still hoping to see the light of the day [11]. AZD1222 (ChAdOxI-S) by Oxford University/AstraZeneca, inactivated Coronavac vaccine (Sinovac), and inactivated vaccines from the Wuhan Institute of the Biological Products and Beijing Institute of Biological Products (Sinopharm) respectively are in the leading front followed by other vaccines like Ad26COVS1 (Janssen pharmaceuticals), GX19 (Genexine consortium), BBV152A (Bharat Biotech), and KBP-COVID-19 (Kentucky Bioprocessing).

\section{Origin of hypothesis}

SARS-CoV-2, primarily a respiratory pathogen, targets alveolar sacs as the main target site. Following infection either through direct contact, aerosols, droplets, or airborne transmission [12], the spike protein exhibits a preferential binding with angiotensin-converting enzyme 2 (ACE2) receptors present on the alveolar cells and gets internalized [13].SARS-CoV-2 single-stranded positive-sense RNA is being transcribed to form its replicative negative-strand through RNA-dependent RNA-polymerase (RdRp). The body responds to the infection by recruiting immune cells at the site of infection thereby leading to the release of proinflammatory cytokines in excess as a hyper response and immune activation state resulting in cytokine release syndrome (CRS) [14]. In return, the significant production of interleukin-6 (IL-6) in the plasma leads to an increase in vascular permeability, acute respiratory distress syndrome (ARDS), and finally death [15]. Generally, IL-6 is considered to be a profibrotic molecule having both pro-and anti-inflammatory properties. Studies suggested that carriers with IL-6 gene polymorphism (IL6 174C allele) have higher severity of pneumonia along with higher IL-6 levels $[16,17]$.

The antivirals under recommendations for COVID-19 sometimes have limitations including low retention at the site of infection, non-specificity, bioavailability, and the lack of suitable mode of administration. Keeping abreast with the available information with a focus to address the challenges ahead, we plan to have a nano-technological intervention towards delivering potent anti-COVID-19 drugs through nebulizers targeting simultaneously on viral RdRp enzyme which serves as an important component of viral replication inside the host cell and IL-6 receptor (IL6) which mediates inflammatory JAK/STAT (Janus kinases/Signal transducer and activator of transcription proteins) pathway, releasing cytokines leading to CRS and further worsening the disease.

It is proposed to adopt repurposing of a suitable $\mathrm{RdRp}$ inhibitor and an immunomodulator together in a single formulation. As favipiravir targets the RdRp enzyme by chain termination and induce lethal mutagenesis, thereby restricts the viral replication and tocilizumab, a recombinant human monoclonal antibody targets IL-6R and inhibits gp130 signal transduction pathway facilitating the neutralization of the IL-6 mediated inflammation. The plan is to deliver the above antivirals in the form of a mucoadhesive nanoformulation through nasal route for effective drug delivery.

Hypothesis In severe COVID-19 cases, the prominent issue is the exaggerated inflammation in addition to the high viral load. Considering the above facts it is proposed to target simultaneously the IL-6R and RdRp enzyme to reduce the inflammation and viral load respectively. It is hypothesized to put favipiravir (RdRp inhibitor) and tocilizumab (Immunomodulator) duo-combination in mucoadhesive peptide-lipid hybrid-based nanoformulation as an effective treatment regimen for serious COVID-19 patients by nebulization (Fig. 1). Drug delivery across mucus membranes is considered to be an effective route of administration owing to their large surface area and would be beneficial in the field of delivery of sensitive biomolecules and for increasing the residence time of therapeutic molecules. Mucoadhesive systems can be further utilized to achieve site-specific delivery of therapeutic molecules [18]. Additionally, muco-adhesiveness increases the adherence to mucin rich glycoprotein for enabling prolonged retention of the drug in the target issues containing mucus layer.

Thus, here mucoadhesive protein/peptide and lipidbased hybrid self-assembled nanostructures would be used for prolonged retention time in the alveolar epithelium/ epithelial cells covered with mucin. Mucoadhesive 


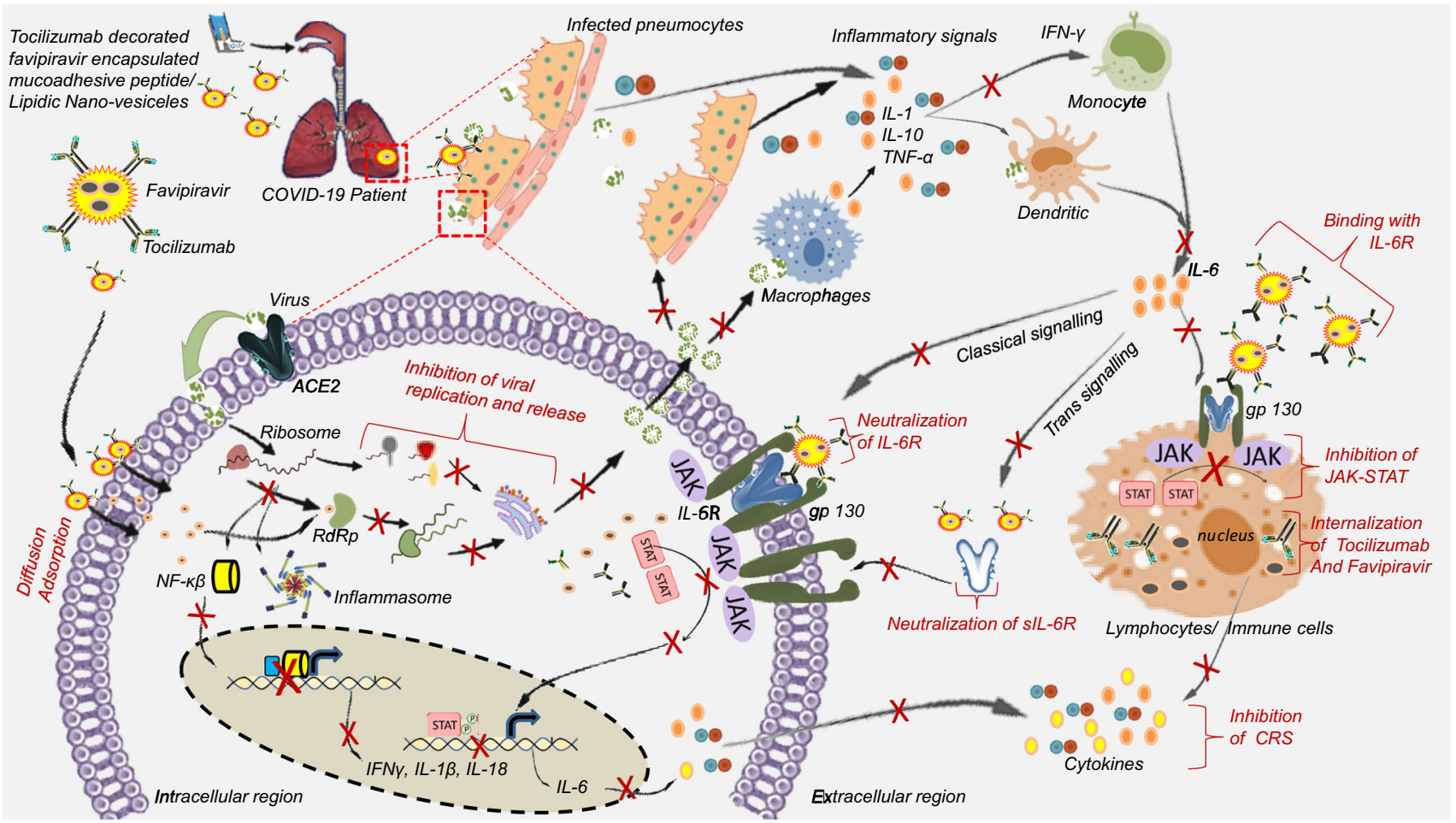

Fig. 1 Graphical presentation of the proposed hypothesis. During infection, SARS-CoV-2 using spike glycoprotein attaches to the ACE2 receptor on the host cell membrane and enters inside the cell where RNA translates to form RdRp, spike, nucleo-capsid, and other proteins, which later assemble and package through the endoplasmic reticulum and Golgi body and finally get released outside the cell as an infectious virion. Excessive damage to host cells results in the immune cell recruitment phenomenon which eventually leads to cytokine release syndrome. Especially IL-6, which binds to the IL-6R and activates the gp130 medicated JAK/STAT pathway. Favipiravir and Tocilizumab co-delivery using mucoadhesive protein-lipidic nanovesicles administered through pulmonary route by nebulizer will

polymers like chitosan, pluronic, hydroxyl propyl methylcellulose in combination with mucoadhesive proteins like sericin, aggregate silk, or designed adhesive peptides like FEFEFKFK will be used to construct the mucoadhesive nanocarriers [19-21]. These can be routed through pulmonary mode using a nebulizer, targeting the lung, which can be non-invasive, provide easy of administration and offer portability. The promising evidence of such concept is the intranasal vaccines like BBV154 and SINO-Vac which will stimulate a broad immune response and neutralize the $\operatorname{IgG}$, and mucosal $\operatorname{IgA}[22]$.

The nanostructures will be further surface decorated with tocilizumab by adopting carbodiimide crosslinker chemistry and the antiviral drug will be loaded inside the nanoparticle core.

This hypothesis has been conceived to explore the application of nanotechnology-based drug formulation which protects the drugs from enzymatic degradation, help in two ways: Mucoadhesiveness would help the nanovesicles to have an extended retention time in the lung tissues. Nanovesicles will facilitate pro-antiviral favipiravir to be delivered inside the infected cells where it gets activated by ribosylation and phosphorylation to active favipiravir-RTP, which subsequently will bind the RdRp enzyme and inhibit viral replication. This will cease virion production. Tocilizumab antibody coated onto the nanovesicles would bind to the soluble IL-6R or IL-6R present on the cell membrane, thereby restricting IL-6 binding with the receptors. This will result in the inhibition of JAK/STAT signaling pathway and simultaneously, reduce the inflammatory cascade reaction

increases tissue absorption, dissolution, and enables controlled drug release at the specific site.

\section{Supportive evidence exploring the concept}

SARS-CoV-2 like Influenza and Ebola is a positive-sense RNA virus, where RdRp plays an important role in viral replication. Favipiravir (T705), a purine nucleoside analog, is being licensed for the treatment of influenza in Japan [23]. Further, favipiravir has been shown to have inhibiting effects on RNA viruses including Ebola, Lassa fever virus, Rabies, Respiratory Syncytial Virus (RSV), Rhinovirus, and Polioviruses in in-vitro as well as in animal experiments [24]. Oseltamivir and zanamivir-resistant influenza $\mathrm{A}$ and $\mathrm{B}$ strains in addition to SARS-CoV-2 are reported to be affected by favipiravir in the in-vitro model [25]. Interestingly, the non-development of resistance to favipiravir, as observed in a mice infection model, has well 
proved its therapeutic role in the treatment of influenza pandemics [26]. The prodrug, favipiravir gets activated to favipiravir ibofuranosyl-5'-triphosphate (T-705-RTP) following intracellular ribosylation as well as phosphorylation [27]. T-705-RTP being purine nucleosides acts as a competitive inhibitor of RdRp, interferes with the viral RNA replication. The drug with a minimum inhibitory concentration (IC50) of $0.022 \mu \mathrm{g} / \mathrm{mL}$, can inhibit influenza virus [28]. The limitation with favipiravir is its short half-life of 2-5.5 $\mathrm{h}$ following oral administration. The drug achieves its maximum plasma concentration at $2 \mathrm{~h}$ following administration [29]

Evidence from a proof-of-concept trial with favipiravir has shown a positive trend toward survival in an outbreak of Ebola in West Africa during 2014-2015 [30]. India has approved favipiravir for being marketed as Fabiflu (200 mg tablet) as the first oral medication, with a claim to reduce the viral load in a period of 4 days. The drug cause up to $88 \%$ reduction in symptoms in mild to moderate COVID19 patients [31].

Tocilizumab is a humanized monoclonal antibody that competitively binds to membrane-attached and soluble forms of IL-6R, thereby impeding the gp130 mediated JAK/STAT signaling pathway. This was approved by Food and Drug Administration (US-FDA) for the management of CRS, believed to be caused due to the massive release of pro-inflammatory cytokines as well as chemokines like IL$1 \beta$, IL-6, IL-18) [14] in response to life-threatening complications of chimeric antigen receptor (CAR-T) based cell therapy. In severe COVID-19 infection, IL-6 upsurge has been linked to CRS [32, 33].

RdRp inhibitors like Remdesivir were approved for COVID-19 under Emergency Use authorization [34]. Various studies suggested the clinical significance of Vitamin D and lactoferrin (NCT04427865) [35] in different formulations like liposomal lactoferrin (NCT04475120) [36] as prophylactic treatment as nutritional supplements in the prevention of COVID-19 [37]. These may enhance and boost immunity and therefore protect against viral infection.

Mimicking the natural route of respiratory viral infection, the pulmonary route of drug administration facilitates the direct contact of the antiviral drug at the target site. This mode of delivery provides high local drug concentration with a collateral augmentation of the pharmacological effect as evident in asthma and chronic obstructive pulmonary disease (COPD). Pulmonary delivery of nanoparticles avoids side effects elicited by parenteral routes of drug administration and provides systemic therapy by tweaking the physiochemical characteristic of drugladen nanoparticles, making them suitable for their absorption through the alveolar epithelium. This concept has been supported by many studies. Salinomycin (SAL) inhibits viral RNA replication and has been suggested to be administered through pulmonary mode by nanostructured lipid carriers (NLCs) for treating SARS-CoV-2 [38]. This may prevent the interaction between SARS-CoV-2 spike protein and ACE2 receptor, thereby inhibiting the release of viral RNA into the cytoplasm [39]. There are accumulating pieces of evidence where inhalation of anti-viral antibodies (e.g., IL-7 Fc) and live-attenuated vaccines as therapeutics are highly effective [40].

The classical example of a successful intranasal live attenuated influenza vaccine is Flumist Quadrivalent, developed by AstraZeneca, which is marketed as an immunoprophylaxis vaccine. So far, no substantial achievement for protein therapeutics given by pulmonary mode has been achieved. However, for COPD exacerbation treatment, IFN-b1a (SNG001) immunostimulant was developed and assessed for efficacy through topical lung delivery in phase II trial (NCT03570359) by Synairgen/ AstraZeneca in 2019 [41]. Thus, respiratory delivery of immunoprophylaxis has been proved to be superior to conventional routes against respiratory viruses.

\section{Concluding remarks}

At present, the Moderna and Pfizer-BioNTech mRNA vaccine for COVID-19 in circulation should address the issue of effectiveness against the new SARS-CoV-2 variants emerging in different geographical locations with the potentiality to have increased transmission, disease severity and mortality, therefore the concept of a novel nanoparticle-based therapeutics delivery system directing a combination of drugs to the infected lung tissues for achieving high therapeutic concentration at the infection site would be a useful preposition towards restricting the COVID-19 pandemic and developing an alternate therapeutic modality.

Acknowledgments Indian Council of Medical Research (ICMR), India provides Junior/Senior Research Fellowship to Vikram Thakur. Jiban Jyoti Panda would like to thank ICMR-DHR for the International Faculty fellowship.

\section{Declarations}

Conflict of interest The authors declare that they have no conflict of interest.

\section{References}

1. Coronavirus update (Live): $112,622,329$ cases and 2,494,704 deaths from COVID-19 virus pandemic-worldometer. [Online]. Available: https://www.worldometers.info/coronavirus/. [Accessed: 24-Feb-2021]. 
2. Petersen E, Koopmans M, Go U, Hamer DH, Petrosillo N, Castelli F, Storgaard M, Al Khalili S, Simonsen L. Comparing SARS-CoV-2 with SARS-CoV and influenza pandemics. Lancet Infect Dis. 2020;20(9):e238-44. https://doi.org/10.1016/S14733099(20)30484-9.

3. Chowell G, Castillo-Chavez C, Fenimore PW, Kribs-Zaleta CM, Arriola L, Hyman JM. Model parameters and outbreak control for SARS. Emerg Infect Dis. 2004;10(7):1258-63. https://doi.org/10. 3201/eid1007.030647.

4. Khan A, Naveed M, Dur-e-Ahmad M, Imran M. Estimating the basic reproductive ratio for the Ebola outbreak in Liberia and Sierra Leone. Infect Dis Poverty. 2015;4(1):13. https://doi.org/10. 1186/s40249-015-0043-3.

5. Cucinotta D, Vanelli M. WHO declares COVID-19 a pandemic. Acta Biomedica. 1885;91(1):157-60. https://doi.org/10.23750/ abm.v91i1.9397.

6. Syal K. COVID-19: Herd immunity and convalescent plasma transfer therapy. J Med Virol. 2020;92(9):1380-2. https://doi.org/ 10.1002/jmv.25870.

7. Kupferschmidt K. WHO launches global megatrial of the four most promising coronavirus treatments. Science. 2020. https:// doi.org/10.1126/science.abb8497.

8. Keech $\mathrm{C}$, et al. Phase 1-2 trial of a SARS-CoV-2 recombinant spike protein nanoparticle vaccine. $\mathrm{N}$ Engl J Med. 2020;383(24):2320-32. https://doi.org/10.1056/ NEJMoa2026920.

9. Powell AE, et al. A single immunization with spike-functionalized ferritin vaccines elicits neutralizing antibody responses against SARS-CoV-2 in mice. bioRxiv. 2020. https://doi.org/10. 1101/2020.08.28.272518.

10. U.S. food and drug administration. [Online]. Available: https:// www.fda.gov/. [Accessed: 24-Feb-2021].

11. Draft landscape and tracker of COVID-19 candidate vaccines" [Online]. Available: https://www.who.int/publications $/ \mathrm{m} / \mathrm{item} /$ draft-landscape-of-covid-19-candidate-vaccines. [Accessed: 24-Feb-2021].

12. Klompas M, Baker MA, Rhee C. Airborne transmission of SARS-CoV-2: theoretical considerations and available evidence. JAMA J Am Med Assoc. 2020;324(5):441-2. https://doi.org/10. 1001/jama.2020.12458.

13. Huang Y, Yang C, Feng Xu X, Xu W, Wen Liu S. Structural and functional properties of SARS-CoV-2 spike protein: potential antivirus drug development for COVID-19. Acta Pharmacol Sin. 2020;41(9):1141-9. https://doi.org/10.1038/s41401-020-0485-4.

14. Liu B, Li M, Zhou Z, Guan X, Xiang Y. Can we use interleukin-6 (IL-6) blockade for coronavirus disease 2019 (COVID-19)-induced cytokine release syndrome (CRS)? J Autoimmun. 2020. https://doi.org/10.1016/j.jaut.2020.102452.

15. Magro G. SARS-CoV-2 and COVID-19: Is interleukin-6 (IL-6) the 'culprit lesion' of ARDS onset? What is there besides Tocilizumab? SGP130Fc. Cytokine. 2020. https://doi.org/10.1016/j. cytox.2020.100029.

16. Ulhaq ZS, Soraya GV. Anti-IL-6 receptor antibody treatment for severe COVID-19 and the potential implication of IL-6 gene polymorphisms in novel coronavirus pneumonia. Med Clin (Barc). 2020;155(12):548-56. https://doi.org/10.1016/j.medcli. 2020.07.002.

17. Kirtipal N, Bharadwaj S. Interleukin 6 polymorphisms as an indicator of COVID-19 severity in humans. J Biomolecular Str Dyn. 2020. https://doi.org/10.1080/07391102.2020.1776640.

18. Takeuchi H, Yamamoto H, Kawashima Y. Mucoadhesive nanoparticulate systems for peptide drug delivery. Adv Drug Deliv Rev. 2001;47(1):39-54. https://doi.org/10.1016/S0169409X(00)00120-4.
19. Kavitha K, Rupesh Kumar M, Jagadeesh Singh S. Novel mucoadhesive polymers-a review. J Appl Pharm Sci. 2011;01(08):37-42.

20. Tang C, Miller AF, Saiani A. Peptide hydrogels as mucoadhesives for local drug delivery. Int J Pharm. 2014;465(1-2):427-35. https://doi.org/10.1016/j.ijpharm.2014.02.039.

21. Brooks AE. The potential of silk and silk-like proteins as natural mucoadhesive biopolymers for controlled drug delivery. Frontiers in Chemistry. 2015;3:65. https://doi.org/10.3389/fchem.2015. 00065.

22. Indian nasal vaccine will be a game-changer against Covid-the sunday guardian live. [Online]. Available: https://www.sunday guardianlive.com/news/indian-nasal-vaccine-will-game-changercovid. [Accessed: 25-Feb-2021].

23. Shiraki K, Daikoku T. Favipiravir, an anti-influenza drug against life-threatening RNA virus infections. Pharmacol and Ther. 2020. https://doi.org/10.1016/j.pharmthera.2020.107512.

24. Oestereich L, Lüdtke A, Wurr S, Rieger T, Muñoz-Fontela C, Günther S. Successful treatment of advanced Ebola virus infection with T-705 (favipiravir) in a small animal model. Antiviral Res. 2014;105(1):17-21. https://doi.org/10.1016/j.antiviral.2014. 02.014 .

25. Abed Y, Boivin G. A review of clinical influenza A and B infections with reduced susceptibility to both oseltamivir and zanamivir. Open Forum Infect Dis. 2017. https://doi.org/10.1093/ ofid/ofx 105 .

26. Furuta Y, et al. T-705 (favipiravir) and related compounds: Novel broad-spectrum inhibitors of RNA viral infections. Antiviral Res. 2009;82(3):95-102. https://doi.org/10.1016/j.antiviral.2009.02. 198.

27. Furuta Y, Komeno T, Nakamura T. Favipiravir (T-705), a broad spectrum inhibitor of viral RNA polymerase. Proc Japan Acad Series B: Phys Biol Sci. 2017;93(7):449-63. https://doi.org/10. 2183/pjab.93.027.

28. Furuta Y, et al. Mechanism of action of T-705 against influenza virus. Antimicrob Agents Chemother. 2005;49(3):981-6. https:// doi.org/10.1128/AAC.49.3.981-986.2005.

29. Coomes EA, Haghbayan H. Favipiravir, an antiviral for COVID19? J Antimicrobial Chemotherapy. 2020;75(7):2013-4. https:// doi.org/10.1093/jac/dkaa171.

30. Sissoko D, et al. Experimental treatment with favipiravir for Ebola virus disease (the JIKI trial): a historically controlled, single-arm proof-of-concept trial in Guinea. PLoS Med. 2016. https://doi.org/10.1371/journal.pmed.1001967.

31. Favipiravir is now the first oral COVID-19 treatment approved in India: all you need to know-Health News, Firstpost. [Online]. Available: https://www.firstpost.com/health/favipiravir-is-nowthe-first-oral-covid-19-treatment-approved-in-india-all-you-needto-know-8509951.html. [Accessed: 24-Feb-2021].

32. Ingraham NE, et al. Immunomodulation in COVID-19. The Lancet Respir Med. 2020;8(6):544-6. https://doi.org/10.1016/ S2213-2600(20)30226-5.

33. Le RQ, et al. FDA approval summary: tocilizumab for treatment of chimeric antigen receptor $t$ cell-induced severe or life-threatening cytokine release syndrome. Oncologist. 2018;23(8):943-7. https://doi.org/10.1634/theoncologist.2018-0028.

34. A. Rhoades (2020) Veklury (remdesivir) EUA letter of approval, reissued 10/22/2020.

35. Utility of lactoferrin as a preventive agent for healthcare workers exposed to COVID-19-full text view-ClinicalTrials.gov. [Online]. Available: https://clinicaltrials.gov/ct2/show/ NCT04427865. [Accessed: 25-Feb-2021].

36. Efficacy and safety of liposomal lactoferrin in COVID-19 patients with mild-to-moderate disease and in COVID-19 asymptomatic patients-full text view-ClinicalTrials.gov. 
[Online]. Available: https://clinicaltrials.gov/ct2/show/ NCT04475120. [Accessed: 25-Feb-2021].

37. Costagliola G, Spada E, Comberiati P, Peroni DG. Could nutritional supplements act as therapeutic adjuvants in COVID-19? Ital J Pediatr. 2021;47(1):32. https://doi.org/10.1186/s13052-02100990-0.

38. Pindiprolu SKSS, et al. Pulmonary delivery of nanostructured lipid carriers for effective repurposing of salinomycin as an antiviral agent. Med Hypotheses. 2020. https://doi.org/10.1016/j. mehy.2020.109858.

39. De Clercq E. Potential antivirals and antiviral strategies against SARS coronavirus infections. Exp Rev Anti-Infect Ther. 2006;4(2):291-302. https://doi.org/10.1586/14787210.4.2.291.
40. Kang MC, et al. Intranasal introduction of fc-fused interleukin-7 provides long-lasting prophylaxis against lethal influenza virus infection. J Virol. 2016;90(5):2273-84. https://doi.org/10.1128/ jvi.02768-15.

41. Monk PD, et al. Safety and efficacy of inhaled nebulised interferon beta-1a (SNG001) for treatment of SARS-CoV-2 infection: a randomised, double-blind, placebo-controlled, phase 2 trial. Lancet Respir Med. 2021;9(2):196-206. https://doi.org/10.1016/ S2213-2600(20)30511-7.

Publisher's Note Springer Nature remains neutral with regard to jurisdictional claims in published maps and institutional affiliations. 